\title{
Acta
Biochimica
Polonica
}

Vol. 48 No. 3/2001

$711-717$

QUARTERLY

This work is dedicated to Professor Jacek Augustyniak

\section{Differences in editing of mitochondrial nad3 transcripts from CMS and fertile carrots}

\author{
Michał Rurek ${ }^{1}$, Marek Szklarczyk ${ }^{2}$, Natalia Adamczyk ${ }^{1}$, Barbara Michalik ${ }^{2}$ \\ and Halina Augustyniak ${ }^{1 凶}$ \\ ${ }^{1}$ Department of Plant Molecular Biology, Institute of Molecular Biology and Biotechnology, \\ Adam Mickiewicz University, Poznań, Poland; ${ }^{2}$ Department of Genetics, Plant Breeding and \\ Seed Science, Cracow Agricultural University, Kraków, Poland
}

Received: 22 January, 2001; revised: 11 April, 2001; accepted: 15 July, 2001

Key words: Daucus carota, cytoplasmic male sterility, nad3 gene, RNA editing

A high level of the nucleotide sequence conservation was found for mitochondrial nad3 gene of carrot. Three silent nucleotide substitutions differentiate nad3 open reading frames from cytoplasmic male sterile and male fertile carrots. All these differences are preserved on the RNA level. Partial and silent editing also distinguished both carrots. Three of the $\mathrm{C}$ to $\mathrm{U}$ conversions were specific to the fertile line. In the two examined carrot lines editing did not affect the mode of alteration of encoded amino acids.

Exciting new findings have emerged as concerns plant mitochondria in relation to the manifestation of cytoplasmic male sterility (CMS) [1, 2]. Molecular studies on CMS have revealed the existence of a rearrangement modification in mitochondrial DNA which, in turn, leads to stamen sterility or pollen abortion in several plant species. An important feature of this rearrangement responsible for CMS was elucidated by the discovery of chimeric genes which are often cotranscribed with other standard mitochondrial genes. CMS provides a convenient means to produce hybrid seeds. Two CMS types are widely used for carrot hybrid seeds production: brown-anther and petaloid sterility [3]. Petaloid plants

\footnotetext{
${ }^{\circledR}$ Corresponding author: H. Augustyniak, Institute of Molecular Biology and Biotechnology, A. Mickiewicz University, 5 Międzychodzka Str., 60-371 Poznań, Poland; e-mail: haaugust@main.amu.edu.pl

Abbreviations: AGPC, acid guanidinium thiocyanate-phenol-chloroform; AMV, avian myeloblastosis virus; CMS, cytoplasmic male sterility; CTAB, hexadecyltrimethylammonium bromide; RT-PCR, reverse transcriptase polymerase chain reaction.
} 
lack stamens which depending on the nuclear background of the sterile cytoplasm have been transformed into petals, petal-like, bract-like or carpelloid structures [4]. The molecular aspects of CMS in carrots have been investigated in several laboratories [5-8]. Recently Szklarczyk et al. [8] reported specific organization and transcription of atp9 gene from petaloid carrot cytoplasm and also speculated that $\mathrm{C}$ to $\mathrm{U}$ conversion in the second glutamine triplet of the atp9 gene extension could play role in fertility restoration. Here, we attempted to test if the type of cytoplasm might influence RNA editing of another carrot mitochondrial gene. We chose nad3 gene for its high content of edited nucleotides. Wilson \& Hanson [9] studied the nad3 transcripts population from petunia CMS lines and showed that the extent of editing at the three assayed positions varied depending on nuclear background of the sterile cytoplasm. However, Hanson et al. [10] have detected no correlation of transcripts editing with either sterility or fertility restoration. These results prompted us to analyse RNA editing in carrot nad3. Previously we examined the sequence and transcription of the $n a d 3$ and rps 12 genes in four lupin species and found that the structure and organization of these genes were conserved [11]. The aim of this study was to analyse genomic structure and degree of RNA editing of the nad3 genes from CMS petaloid and fertile carrot cytoplasm.

\section{MATERIALS AND METHODS}

Plant material. Carrot inbred lines: 2163A (petaloid CMS) and 2163B (maintainer) were provided by Production and Breeding of Horticultural Plants Ltd. (PHRO, Krzeszowice, Poland).

Isolation of nucleic acids. Total cellular DNA for the polymerase chain reaction (PCR) was extracted from young leaves according to Gawal \& Jarret [12] with an additional chloroform extraction step included. Prior to this step the aqueous phase collected from the first extraction was supplemented with 0.1 vol. of $10 \%$ CTAB, $0.7 \mathrm{M} \mathrm{NaCl}$ solution. Carrot mitochondrial DNA (mtDNA) was isolated from root tissue using the procedure of Steinborn $e t$ al. [13]. The ammonium acetate precipitation step was omitted. Plasmid DNA to be used as a sequencing template was isolated with Qiagen Miniprep Kit. PCR fragments to be used for subsequent cloning and sequencing were purified using Qiaex II Gel Extraction Kit (Qiagen). Total cellular RNA was extracted from young umbels using the modified AGPC method. After the first isopropanol precipitation described in the original procedure [14], the RNA pellet was re-hydrated and lithium chloride re-precipitated [15].

DNA cloning. The mtDNA library of petaloid line 2163A was constructed in Bluescript II KS phagemid vector (Stratagene) as described by Szklarczyk et al. [8]. The resulting library was screened with nad3 probe from 2163A line, labelled as described by Rurek et al. [11]. The insert size from two positive clones was determined using BamHI digestion.

The PCR amplified nad3 genes were cloned into pGEM T-Easy vector (Promega) according to procedure of manufacturer's recommendations.

PCR amplification of DNA. The PCR reaction mixture contained in $50 \mu \mathrm{l}$ the following components: $60 \mathrm{ng}$ of total DNA, $0.45 \mu \mathrm{M}$ of each primer, $10 \mathrm{mM}$ Tris $/ \mathrm{HCl}, \mathrm{pH} 8.8,1.5 \mathrm{mM}$ $\mathrm{MgCl}_{2}, 50 \mathrm{mM} \mathrm{KCl}, 0.1 \%$ (v/v) Triton X-100, $200 \mu \mathrm{M}$ of each dNTP, 0.03 units of PrimeZyme polymerase (Biometra) per microlitre of the reaction mixture. Five synthetic primers: P1, P2, P3, P4, P5 specific to the angiosperm consensus DNA fragment including the nad3 gene alone or nad3 with flanking sequences were used. PCR was carried out in a Biometra 050-500 cycler. For the above primers one step amplification was used: the initial denaturation for $95^{\circ} \mathrm{C}$ for 5 min was followed by 30 cycles of $45 \mathrm{~s}$ at $92^{\circ} \mathrm{C}, 30 \mathrm{~s}$ at $57^{\circ} \mathrm{C}$ and $2 \mathrm{~min}$ at $72^{\circ} \mathrm{C}$. The PCR 
was terminated by elongation cycle of $10 \mathrm{~min}$ of $72^{\circ} \mathrm{C}$.

List of primers. Oligonucleotides used for PCR, sequencing and reverse transcription:

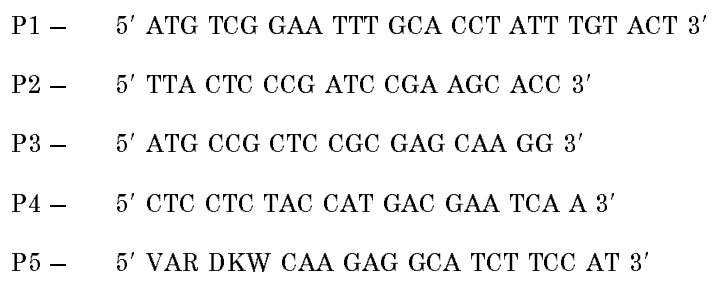

DNA sequencing. This was performed by the dideoxy chain termination method [16] using either Perkin Elmer (ABI Prism BigDye Terminator Cycle Sequencing Kit) or Amersham-Pharmacia Biotech (Thermo Sequenase Fluorescent ( ${ }^{*} \mathrm{Cy} 5,5$ ' end) Labelled Primer Cycle Sequencing Kit with 7-deaza-dGTP) and P1, P3 and P4 primers. Sequencing reactions were set up and cycled according to the manufacturer's recommendations. Reaction products were resolved and analysed using either the AbiPrism 377 DNA Sequencer or Pharmacia-Amersham ALF Sequencer.

Reverse transcription of RNA. The cDNA from six separate RT-PCR runs was synthesized from total RNA of carrot lines 2163A and 2163B and specific primer P5 by AMV reverse transcriptase (Promega) following the Promega protocol. For the reaction $4 \mu \mathrm{g}$ of total RNA, $0.5 \mu \mathrm{g}$ of P5 primer per $\mu \mathrm{g}$ of RNA and 15 units of AMV reverse transcriptase were used. The cDNA synthesis was terminated by phenol/chloroform extraction. The RT products were ethanol precipitated, dissolved in water and used for amplification.

\section{RESULTS AND DISCUSSION}

\section{The nad 3 gene from petaloid and fertile carrot cytoplasm}

In order to isolate carrot nad3 gene, a genomic library of mt DNA from 2163A line was screened with PCR fragment amplified from petaloid carrot DNA using P1 and P2 primers. Two positive clones were identified with insert sizes of 4.5 and $17 \mathrm{~kb}$. Restriction mapping and hybridization analysis (not shown) of these clones confirmed that they contained the nad3 gene. Sequence analysis of the $4.5 \mathrm{~kb}$ clone revealed a 357-nucleotide open reading frame (ORF) identified by similarity as the nad3 gene: the nad $3 \mathrm{ORF}$ showed more than $93 \%$ sequence identity with the respective sequences of dicotyledons and about $90 \%$ identity with other angiosperms (GenBank data). Downstream from the nad3 ORF, a coding sequence of rps 12 gene is located. The spacer region between these ORFs has 48 nucleotides.

Once the sequence of nad 3 gene from the petaloid line was determined it was possible to perform PCR amplification of this gene with specific primers using a genomic template from the same line. Both genomic (from the library) and amplified genomic sequences were identical proving that in petaloid carrot nad3 sequence does not exhibit heteroplasmy.

PCR amplification was also used to determine the sequence of nad3 gene from fertile carrot. Comparison of the sequence of nad3 genes from the two kinds of cytoplasm revealed three single-nucleotide substitutions within a DNA segment of nad3 ORFs and its flanking regions including 25 nucleotides at $5^{\prime}$ end, 48 nucleotides of the spacer and $18 \mathrm{nu}-$ cleotides of the rps 12 ORFs. Differentiating nucleotides are located in the following positions of the nad3 genes: 15th, 105th and 312th (EMBL accession numbers: AF285878, AF285879). In the case of the fertile line they contain $\mathrm{A}, \mathrm{T}$ and $\mathrm{T}$, while the sequence of petaloids has G, C and C, respectively. No sequence differences were observed when the same fragment of another clone from petaloid line was analysed. All substitutions appeared in the third codon position and therefore the deduced amino-acid sequences of NAD3 proteins remained identical.

The conserved arrangement of carrot nad3 and a part of rps 12 sequences suggest their be- 
longing to one transcription unit. To establish their cotranscription, an RT-PCR experiment was performed with two primers: P5 and P3. The P5 primer corresponds to the 3 ' flanking region of the rps 12 gene, while the $\mathrm{P} 3$ primer to $5^{\prime}$ flanking region of the nad3 gene. Both for fertile and CMS carrots PCR yielded a product of about $780 \mathrm{bp}$, strongly suggesting cotranscription of nad3 and rps 12 (Fig. 1). within the $5^{\prime}$ part of rps 12 gene (not shown). Most of the editing sites are shared between the two analysed carrot lines. Positions 43, 89, and 102 are edited only in fertile carrot, and these positions are located in codons 15 , 30 and 34 (Table 1). The fertile carrot also exhibits the presence of partial editing in codons 15, 30, 34 and 77 (Table 1). The partial editing of codon 15 concerns the first letter of

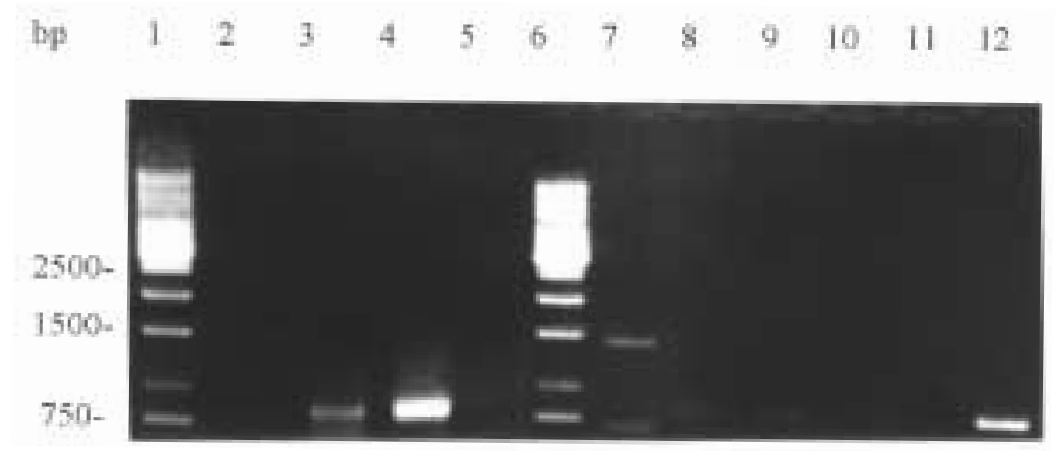

Figure 1. RT-PCR analysis of the cotranscription of nad3 and rps 12 genes in CMS and fertile carrots.

Lanes 1,6 and 7: molecular mass marker. Lane 3: the product of RT-PCR obtained by amplification with P3 and P5 primers in 2163B fertile line. Lane 4: the positive control of PCR (nad3-rps12 genes amplified with P3 and P5 primers). Lanes 8 and 9: the product of RT-PCR obtained by amplification with P3 and P5 primers in 2163A sterile line. Lane 12: the positive control of PCR. Lanes 5 and 11: negative control of amplification without RNA. Lanes 2 and 10: negative control of amplification without AMV reverse transcriptase.

The length of the product confirms that organization of the nad3-rps 12 transcription unit in carrots follows the conserved pattern found in other plant species [9, 17].

\section{Editing of the nad 3 transcripts in petaloid and fertile carrots}

To study the extent of RNA editing, the PCR derived cDNAs of nad 3 transcripts of the two carrot lines studied were cloned and sequenced. Comparison of genomic and cDNA sequences revealed 19 editing sites - all of them of $\mathrm{C}$ to $\mathrm{U}$ type commonly found in plant mitochondria [18] (EMBL accession number: AJ300556). The nucleotide positions of these sites are the following: $5,39,43,44,61,62,89$, 102, 208, 209, 215, 230, 231, 247, 266, 275, 317, 344 and 349. Another editing site was found 15 nucleotides upstream from nad3 ORF. The last $\mathrm{C}$ to $\mathrm{U}$ conversion was observed the codon. The second letter of this codon is edited both in the sterile and fertile lines. In the case of codon 77, partial editing concerns the third letter. The presence of partial editing in the fertile line was not fully investigated, because only three clones of this line

Table 1. Differences in editing of some codons in the nad3 transcripts from sterile $2163 \mathrm{~A}$ and fertile 2163B line of carrot

\begin{tabular}{lll}
\hline $\begin{array}{l}\text { Codon } \\
\text { number }\end{array}$ & $\begin{array}{l}\text { Codon changes } \\
\text { carrot line 2163A }\end{array}$ & $\begin{array}{l}\text { Codon changes } \\
\text { carrot, line 2163B }\end{array}$ \\
\hline 15 & CCG-CUG (P-L) & CCG-UUG (P-L)* \\
30 & UCC (S) & UCC-UUC (S-F)*1 \\
34 & ACC (T) & ACC-ACU (T) ${ }^{* 1}$ \\
77 & UCC-UUU (S-F) & UCC-UUU $(\mathbf{S}-\mathbf{F})^{*}$ \\
\hline
\end{tabular}

Edited nucleotides and the corresponding amino-acid changes are indicated by bold letters. A star (*) indicates the presence of partial editing of clones of the fertile line. ${ }^{1}$ indicates the presence of unedited codons in part of transcripts of fertile line. 
were analysed. No cases of partial editing were observed for three analysed clones of petaloid line 2163A, although we cannot exclude that they could have been detected if a larger number of cDNA molecules were checked. Lu and Hanson [19] distinguished three categories of transcripts with respect to the editing extent. Our results indicate that carrot nad3 mRNA might belong to the second category transcripts, which are heteroge- neous in editing extent and in which partially edited transcripts are readily detectable. It is interesting to note that nad3 transcripts of petunia and Oenothera also belong to this category [19].

In both carrot lines editing of the nad3 transcripts results in replacement of four prolines by leucines and one proline by phenylalanine. Three serine codons are converted into phenylalanine triplets and in one case trypto-

Table 2. Comparison of amino-acid changes resulting from codon editing of carrot mitochondrial nad3 transcripts with nad 3 transcripts of other higher plants

\begin{tabular}{|c|c|c|c|c|c|c|c|c|c|c|c|c|c|c|}
\hline 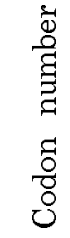 & $\begin{array}{l}\frac{\pi}{0} \\
\stackrel{0}{0} \\
\stackrel{\tilde{\Xi}}{0} \\
\dot{\theta}\end{array}$ & 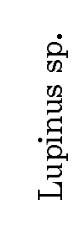 & 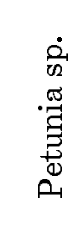 & 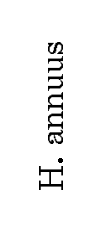 & 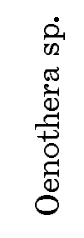 & 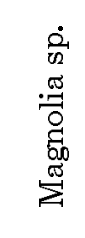 & 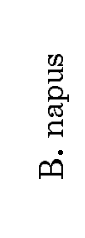 & $\begin{array}{l}\text { 吅 } \\
\stackrel{d}{0} \\
\dot{4}\end{array}$ & 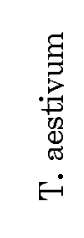 & $\begin{array}{l}\tilde{0} \\
\frac{0}{0} \\
.0 \\
.0 \\
\dot{0}\end{array}$ & 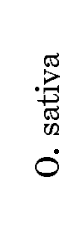 & 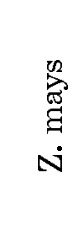 & 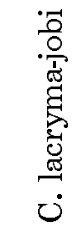 & 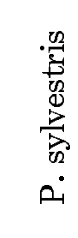 \\
\hline 2 & S-L & S-L & S-L & S-L & S-L & S-L & S-L & S-L & T-L & & & S-L & S-L & \\
\hline 13 & I & & & & & & & & I & & & & & \\
\hline 15 & P-L & & P-L & P-L & P-L & P-L & & P-L & P-L & P-L & P-L & P-L & P-L & S-L \\
\hline 21 & P-L & P-L & P-L & P-L & & P-L & $\mathrm{L}$ & P-L & P-L & P-L & P-L & P-L & P-L & \\
\hline 27 & & P-L & & P-L & & P-L & P-L & P-L & P-L & P-L & P-L & P-L & P-L & P-L \\
\hline 30 & S-F & & & & & & & & & & & & & P-S \\
\hline 34 & $\mathrm{~T}$ & & & $\mathrm{~T}$ & & & & & & & & & & \\
\hline 49 & & & & & & & & & & & & & S-F & \\
\hline 62 & & & & & & & & & & & & & P-L & \\
\hline 64 & & & & & & & & & & & & & P-S & \\
\hline 70 & $\mathrm{P}-\mathrm{F}$ & $\mathrm{P}-\mathrm{F}$ & $\mathrm{P}-\mathrm{F}$ & P-F & P-F & $\mathrm{P}-\mathrm{F}$ & P-F & P-F & P-F & $\mathrm{P}-\mathrm{F}$ & & $\mathrm{P}-\mathrm{F}$ & P-F & S-F \\
\hline 72 & P-L & P-L & P-L & P-L & P-L & P-L & & P-L & P-L & P-L & & P-L & P-L & P-L \\
\hline 77 & S-F & S-F & S-F & S-F & & S-F & & S-F & S-F & S-F & & S-F & S-F & S-F \\
\hline 83 & P-S & P-S & P-S & P-S & P-S & P-S & P-S & P-S & P-S & P-S & & P-S & P-S & P-S \\
\hline 84 & & P-L & P-L & & L-F & P-L & P-L & P-L & P-L & -L & & P-L & P-L & \\
\hline 89 & P-L & P-L & P-L & P-L & P-L & P-L & & P-L & & & & $\mathrm{L}$ & & P-L \\
\hline 92 & S-F & S-F & S-F & S-F & S-F & S-F & & S-F & S-F & & & S-F & S-F & S-F \\
\hline 106 & S-F & S-F & S-F & S-F & S-F & S-F & & S-F & S-F & S-F & & & S-F & \\
\hline 115 & S-L & S-L & S-L & S-L & S-L & S-L & S-L & S-L & S-L & S-L & & S-L & S-L & S-L \\
\hline 117 & R-W & R-W & R-W & R-W & R-W & R-W & R-W & R-W & R-W & R-W & & R-W & R-W & R-W \\
\hline
\end{tabular}

Abbreviations: D. carota, Daucus carota; H. annuus, Helianthus annuus; B. napus, Brassica napus; A. cepa, Allium cepa; T. aestivum, Triticum aestivum; S. bicolor, Sorghum bicolor; O. sativa, Oryza sativa; Z. mays, Zea mays; C. lacryma-jobi, Coix lacryma-jobi; P. sylvestris, Pinus sylvestris. The data used in this Table are adapted from the following references: Petunia sp. [9], H. annuus [17], Oenothera sp. [21], Magnolia sp. [17], A. cepa [17], B. napus [22], T. aestivum [20], S. bicolor [23], O. sativa [24], Z. mays [25], C. lacryma-jobi [26], P. sylvestris [27]. 
phan replaces arginine (Table 2). In neither of the two examined carrot lines editing does affect the mode of alteration of the encoded amino acids (Tables 1 and 2).

The characteristic feature of the carrot transcripts is the editing resulting in silent codon alterations. We observed two codon changes corresponding to silent events. In the case of fertile carrot, they were found in codons 13 and 34 , for the petaloid carrot only in codon 13 (Tables 1 and 2).

Data collected in Table 2 show that most of the edited codons are evolutionarily conserved in all angiosperms. It is interesting to note that two silent changes which appeared in codons 13 and 34 of carrot are very rare among plants. The peculiar feature of RNA editing of the nad3 transcripts, is the preediting of codon 84 in carrot, sunflower and wheat in spite of the overall panediting of this triplet in the rest of plants [17, 20].

Generally it seems that a part of differences in genomic sequences between all the investigated species are minimized by editing of their mRNAs.

Differential editing of the nad3 transcripts in fertile and CMS carrots raises the possibility that regulation of the extent of RNA editing could generate multiple forms of NAD3 gene products. In this study we have demonstrated that, in carrot mitochondria, the partially edited codon 30 could eventually lead to the change of one amino-acid residue in the protein sequence.

The presented data report for the first time that petaloid and fertile carrots differ in the nucleotide sequence and editing of RNA of nad3 gene.

\section{R E F E R E N C E S}

1. Sarria, R., Lyznik, A., Vallejos, C.E. \& Mackenzie, S.A. (1998) A cytoplasmic male sterility-associated mitochondrial peptide in common bean is post-translationally regulated. Plant Cell 10, 1217-1228.
2. Nakajima, Y., Yamamoto, T., Muranaka, T. \& Oeda, K. (2001) A novel orfB-related gene of carrot mitochondrial genomes that is associated with homeotic cytoplasmic male sterility (CMS). Plant Mol. Biol. 46, 99-107.

3. Stein, M. \& Nothnagel, T. (1995) Some remarks on carrot breeding (Daucus carota sativus Hoffm.). Plant Breed. 114, 1-11.

4. Kitagawa, J., Posluszny, U., Gerrath, J.M. \& Wolyn, D.J. (1994) Developmental and morphological analyses of homeotic cytoplasmic male sterile and fertile carrot flowers. Sex Plant Reprod. 7, 41-50.

5. Bowes, C.E. \& Wolyn, D.J. (1998) Phylogenetic relationships among fertile and petaloid male-sterile accessions of carrot, Daucus carota L. Theor. Appl. Genet. 96, 928-932.

6. Scheike, R.G.E., Brennicke, A. \& MehringLemper, M.G. (1992) Unique patterns of mitochondrial genes, transcripts and proteins in different male-sterile cytoplasms of Daucus carota. Theor. Appl. Genet. 83, 419-427.

7. Szklarczyk, M. (1997) Unique features of carrot mtDNAs from CMS and maintainer lines. J. Appl. Genet. 38A, 42-56.

8. Szklarczyk, M., Oczkowski, M., Augustyniak, H., Boerner, T., Linke, B. \& Michalik, B. (2000) Organisation and expression of mitochondrial atp9 genes from CMS and fertile carrots. Theor. Appl. Genet. 100, 263-270.

9. Wilson, R.K. \& Hanson, M.R. (1996) Preferential RNA editing at specific sites within transcripts of two plant mitochondrial genes does not depend on transcriptional context or nuclear genotype. Curr. Genet. 30, 502-508.

10.Hanson, M.R., Wilson, R.K., Bentolila, S., Kohler, R.H. \& Chen, H.C. (1999) Mitochondrial gene organization and expression in petunia male fertile and sterile plants. J. Hered. 90, 362-368.

11. Rurek, M., Oczkowski, M. \& Augustyniak, H. (1998) Conservation of the structure and orga- 
nization of lupin mitochondrial nad3 and rps12 genes. Acta Biochim. Polon. 45, 695699.

12. Gawal, N.J. \& Jarret, R.L. (1991) A modified CTAB DNA extraction procedure for Musca and Ipomea. Plant Mol. Biol. Rep. 9, 262-266.

13. Steinborn, R., Weihe, A. \& Boerner, T. (1992) Mitochondrial genome diversity within a cultivar of Daucus carota (ssp. sativus) revealed by restriction analysis of single plants. Plant Breed. 109, 75-77.

14. Chomczynski, P. \& Sacchi, N. (1987) Single-step method of RNA isolation by acid guanidinium thiocyanate-phenol-chloroform extraction. Anal. Biochem. 2, 156-159.

15. Gelvin, S.B., Schilperoort, R.A. \& Verma, M. (1991) Plant Molecular Biology Manual. Kluwer Academic Publ., Dordrecht, The Netherlands.

16. Sanger, F., Nickeln, S. \& Coulson, A.R. (1979) DNA sequencing with chain terminating inhibitors. Proc. Natl. Acad. Sci. U.S.A. 74, 5463-5467.

17. Perotta, G., Regina, T.M.R., Ceci, L.R. \& Quagliariello, C. (1996) Conservation of the organization of the mitochondrial nad3 and rps 12 genes in evolutionarily distant angiosperms. Mol. Gen. Genet. 251, 326-337.

18. Maier, R.M., Zeltz, P., Kossel, H., Bonnard, G., Gualberto, J. \& Grienenberger, J.-M. (1996) RNA editing in plant mitochondria and chloroplasts. Plant Mol. Biol. 32, 343-365.

19. Lu, B. \& Hanson, M.R. (1996) Fully edited and partially edited nad 9 transcripts differ in size and both are associated with polysomes in potato mitochondria. Nucleic Acids Res. 24, 1369-1374.

20. Gualberto, J.M., Bonnard, G., Lamattina, L. \& Grienenberger, J.-M. (1991) Expression of the wheat mitochondrial nad3-rps 12 transcription unit: correlation between editing and mRNA maturation. Plant Cell 3, 1109-1120.

21. Schuster, W., Wissinger, B., Unseld, M. \& Brennicke, A. (1990) Transcripts of the NADH-dehydrogenase subunit 3 gene are differentially edited in Oenothera mitochondria. EMBO J. 9, 263-269.

22. Itani, K. \& Handa, H. (1998) Rapeseed mitochondrial ccb206, a gene involved in cytochrome $c$ biogenesis, is co-transcribed with the nad3 and rps 12 genes; Organization, transcription, and RNA editing of the nad3/rps12/ ccb206 locus. Curr. Genet. 34, 318-325.

23. Howad, W. \& Kempken, F. (1997) Sequence analysis and transcript processing of the mitochondrial nad3-rps12 genes from Sorghum bicolor. Plant Sci. 129, 65-68.

24. Suzuki, T., Kazama, S., Hirai, A., Akihama, T. \& Kadowaki, K. (1991) The rice mitochondrial nad3 gene has an extended reading frame at its $5^{\prime}$ end: Nucleotide sequence analysis of rice trnS, nad3, and rps12. Curr. Genet. 20, 331337.

25. Grosskopf, D. \& Mulligan, R.M. (1996) Developmental- and tissue-specifity of RNA editing in mitochondria of suspension-cultured maize cells and seedlings. Curr. Genet. 29, 556-563.

26. Dias, S.M.G., Siquera, S.F., Lejeune, B. \& de Souza, A.P. (2000) Identification and characterization of the trnS/pseudo-tRNA/nad3/ rps 12 cluster from Coix lacryma-jobi L.: Organization, transcription and RNA editing. Plant Sci. 158, 97-105.

27. Karpinska, B., Karpinski, S. \& Hallgren, J.-E. (1995) The genes encoding subunit 3 of NADH dehydrogenase and ribosomal protein S12 are co-transcribed and edited in Pinus sylvestris (L.) mitochondria. Curr. Genet. 28, 423-428. 